\title{
Une ville à refaire. Renouvellement urbain à Belgrade
}

A New Deal for a Town. Urban Renewal in Belgrade

Eva Vaništa Lazarević

\section{(2) OpenEdition}

Journals

Édition électronique

URL : http://journals.openedition.org/etudesbalkaniques/245

ISSN : 2102-5525

Éditeur

Association Pierre Belon

Édition imprimée

Date de publication : 1 janvier 2007

Pagination : 187-198

ISBN : 978-2-910-860-07-8

ISSN : $1260-2116$

\section{Référence électronique}

Eva Vaništa Lazarević, « Une ville à refaire. Renouvellement urbain à Belgrade », Études balkaniques [En ligne], 14 | 2007, mis en ligne le 01 avril 2010, consulté le 07 mai 2019. URL : http:// journals.openedition.org/etudesbalkaniques/245

Ce document a été généré automatiquement le 7 mai 2019.

Tous droits réservés 


\title{
Une ville à refaire. Renouvellement urbain à Belgrade
}

A New Deal for a Town. Urban Renewal in Belgrade

\author{
Eva Vaništa Lazarević
}

\section{Introduction}

\section{Cadre social}

1 La lutte contre la pauvreté et le chômage, la protection des ressources naturelles et de l'environnement représentent les priorités des Nations Unies. La pauvreté de plus en plus grave dans le monde et particulièrement en Serbie est due à la répression économique résultant du chômage et du travail mal rémunéré. Sur le marché du travail se sont établies de nouvelles relations d'offre et de demande issues de la période de transition et de la nouvelle valorisation de la main-d'œuvre. Personne n'a plus besoin de groupes entiers de la population. Le rôle correctif de l'État manque qui engagerait des moyens financiers pour des groupes particuliers (médecins et professeurs) indispensables pour assurer le progrès social. Ainsi pourrait-on exterminer l'économie grise et la corruption, un des plus graves problèmes actuels. Les petites entreprises et le secteur tertiaire, qui représentent une ligne directrice pour la sauvegarde de la paix sociale, ne trouvent plus de clients solvables pour leurs produits et leurs services. Le but du nouveau développement économique comprend l'augmentation des embauches et une hausse des salaires ${ }^{1}$.

2 Belgrade, le croisement-clef de l'Europe au confluent de la plus grande rivière balkanique et du fleuve européen depuis sa genèse n'a jamais connu de période de paix qui ait dépassé 23 années consécutives entre deux guerres et a subi 92 sièges. Suite à la concentration des guerres et à la destruction, la ville a été endommagée à tel point qu'il manquait toujours une période de paix suffisamment longue pour permettre la reconstruction complète de la ville. Dans ce sens, au cours des siècles, la situation 
géopolitique ne se prêtait favorablement ni au progrès ni au développement harmonieux. Ce n'est qu'après 1945, qu'a eu lieu une période d'une quarantaine d'années de paix. C'est ainsi que dans les années 80 du siècle précédent Belgrade était considérée comme une des capitales balkaniques les plus développées, un des centres culturels d'Europe, centre de manifestations internationales extraordinaires, centre des progrès en économie et en industrie, connu également pour ses festivals et ses concerts.

3 Les habitants des Balkans connus pour leur hospitalité, leur humour et leur sensibilité sociale sont, après 15 ans de répression et de guerre, dans les années 1990, économiquement ruinés, dégradés et introvertis. Selon les statistiques de 2002, un tiers de la population est au-dessous du minimum vital. Cependant, les pauvres sont nécessaires à ceux qui en ont moins. La question sociale gagne en priorité et la lutte pour la restitution de la solidarité bat son plein. Des processus graves de réforme et de transition se réalisent en présence d'un phénomène social, inquiétant - la pauvreté.

4 Le régime totalitaire, le pillage des biens publics et la transition à laquelle on est assujetti en Serbie à partir des années 90 ont eu pour conséquence la création d'une nouvelle classe d'oligarques riches, alors que le reste de la population est défavorisé. Le capital social qui se privatise (transition) cause de graves changements sociaux.

5 Au cours du processus de changement de propriétaire des moyens de production, le nombre d'acheteurs potentiels du pays étant insignifiant, c'est vers les étrangers qu'on s'est orienté. Le flux du capital étranger est conditionné par la base du système bancaire ainsi que par la politique financière et monétaire. Le pays appauvri qui s'appuie sur les donations étrangères n'est qu'une stratégie de courte durée et de ce fait il ne reste qu'à s'orienter vers le marché national du capital.

6 Contrairement à la Serbie, dans le cadre du progrès économique, l'Europe s'est occupée des problèmes sociaux en continu (à partir des années 80 jusqu'à présent). En Serbie, on distingue deux périodes de politique sociale. La première, de 1957 à 1985, et la seconde de 1986 jusqu'à présent. Les priorités des politiques sociales ont évolué avec l'évolution des problèmes économiques, politiques et sociaux. Au début, les syndicats s'occupaient de la hausse du niveau de vie, la défense d'embaucher les enfants, l'amélioration des conditions de vie, plus tard des problèmes du cours libre de la main d'œuvre, du traitement égal à l'embauche, puis la décroissance économique a imposé des problèmes complexes au traitement des emplois et des salariés. L'Union européenne peut influencer la politique sociale par sa propre régulation, mais aussi en limitant cette politique dans les pays membres (exemple de la Norvège) ${ }^{2}$.

7 La création du Fonds social européen a visé à réorganiser l'embauche et le maintien des emplois. Il s'agit surtout de chômage à long terme, des femmes qui ont cessé le travail et reviennent travailler de nouveau, des personnes handicapées cherchant du travail, des ouvriers qui ont besoin d'une requalification en raison des progrès technologiques. La charte sociale devrait accompagner le document de Maastricht, mais n'a pas été signée à cause du refus de la Grande-Bretagne. Depuis 1986, la Charte est appliquée en Europe, l'année suivante avec l'avènement du Labour en Grande-Bretagne également. Elle insiste sur la justice sociale, donc sur une loi favorisant le modèle social. Y sont incorporées les améliorations dans le secteur de la santé publique, des conditions de travail, d'information et de consultation des ouvriers, l'égalité des sexes, ainsi que l'intégration des chômeurs et la protection des ouvriers. De nombreux syndicats des pays membres de l'Union ont eu comme motivation de résoudre les problèmes économiques causés par une régulation de production excessive et par leurs résultats inférieurs en Europe par rapport 
aux Etats-Unis et aux pays d'Orient. Ce n'était qu'une tentative, car dans le cas où l'on invente des affaires, la répartition de la plus value n'est que temporaire.

8 Cependant, le modèle des pays plus développés que l'on pourrait suivre se réfère à la socialisation de certaines lois jusqu'à présent 'mercantiles' qui ont été acceptées en Serbie à bras ouverts.

\section{Situation sociale en Serbie}

En Serbie, parallèlement à l'écroulement économique, la guerre civile décennale a saccagé le pays, provoqué une pauvreté plus grave, des réfugiés et des invalides de guerre. Le cadre social est caractérisé par :

- les conséquences du régime totalitaire et le pillage des biens publics,

- les guerres,

- la transition,

- la récession économique.

10 Après l'hyperinflation de 1993 le salaire réel en Serbie (après les hausses et les décroissances par rapport à une meilleure époque (1989)) s'élève à 250 euros par mois. Le secteur privé est de 7 \% supérieur à celui de 1990 mais ne peut pas compenser le licenciement en masse du secteur public qui a dépassé $100 \%$ au cours de 10 années $^{3}$. Ce secteur social représente la génération sacrifiée, le surplus technologique constant.

11 L'assurance sociale devrait disposer de fonds autonomes et doit viser les catégories réellement nécessiteuses ${ }^{4}$. La lutte pour les droits de l'homme, le droit à l'abri, devrait être une priorité non contestée. Le cadre social est indispensable à titre de donnée de base permettant de comprendre la situation urbanistique de Belgrade, qui est extrêmement défavorable et résulte des paramètres sociaux cités.

À partir de 1990 en Serbie le bâti est endommagé pour les raisons suivantes ${ }^{5}$ :

- au moins 600.000 réfugiés et personnes déplacées sont arrivés dans les villes de Serbie,

- la privatisation des logements du secteur public n'a pas amélioré le statut des sans-abri,

- la crise économique et la baisse de niveau de vie ont stoppé le maintien et la reconstruction des immeubles,

- un certain nombre de maisons a été détruit par le bombardement en 1999,

- la construction en baisse constante,

- la construction illégale également en baisse (la loi sur la légalisation a été promulguée pour assurer la paix sociale).

13 Selon les données de novembre 2004, sur les 377.131 réfugiés, 7.000 sont logés dans des centres collectifs ${ }^{6}$. Cependant, en dehors des catégories citées ou dans les catégories des nécessiteux et marginales il y a des personnes âgées, des mères célibataires, des familles avec des membres handicapés ainsi que des personnes excessivement pauvres.

En été 2005 on comptait 60.000 bénéficiaires de l'aide sociale - des invalides de guerre, y compris les membres les plus proches de leur famille ainsi que les familles des soldats disparus. La plupart sont des vétérans des anciennes guerres, 15.000 datent de la dernière guerre. Jusqu'à présent on a accordé la priorité aux problèmes des réfugiés et des Roms aussi bien que des groupes les plus menacés. Les donations les plus importantes sont celles de l'ONU - HABITAT et du Gouvernement italien, Projet de SIRP - logements et intégration des réfugiés en Serbie. L'agence suisse pour le développement et la coopération et l'administration de la ville de Belgrade, qui a initié un programme de 5.000 
logements destinés aux Roms et a assuré 11,5 mil. D'euros. Le Commissariat pour les réfugiés et l'UNR, les "Suisse Disaster Reliefs », le Gouvernement norvégien et plusieurs agences $\mathrm{ONG}$ ont également contribué et amélioré cette situation grave.

L'hébergement des sans-abri est l'une des taches fondamentales pour aider les nécessiteux. En plus, ce groupe manque de confiance en soi-même. Le remède pour amortir ce facteur psychologique important est d'engager ces personnes a exécuter des travaux plus faciles. Ceci exige une stratégie élaborée, une organisation et une coopération des services différents, des pouvoirs locaux, de l'Etat, des organisations internationales ainsi que le financement des banques; dans les pays plus développés, tout un mécanisme d'entreprises, de charges et de responsabilité des participants dans le processus de l'aide sociale au niveau global. Une juridiction adéquate à orientation sociale représente un maillon vers le but final. La Serbie a compris selon sa propre expérience quel rôle joue la solidarité pour résoudre les problèmes sociaux et économiques.

\section{Le développement durable : à quel point est-il praticable en Serbie?}

16 Quand la Conférence des Nations Unis a eu lieu à Rio de Janeiro en 1992 consacrée à la sauvegarde de l'environnement et au développement (UNCED) connue comme la Conférence au Sommet du Globe, personne n'a pu envisager quelles conséquences cette manifestation aurait sur le développement du monde moderne. Ce n'est pas par hasard que la Conférence a eu lieu au Brésil, un des pays évidemment les plus pauvres du monde, avec 7.000 représentants des médias, 116 hommes politiques et 10.000 représentants officiels de 150 pays et une grande tension concernant cet événement. Un échec plus ou moins envisagé à résoudre tous les problèmes de l'univers a suivi ${ }^{7}$. Cependant, plusieurs documents importants ont été adoptés et des processus établis lesquels, en dépit de leur caractère bureaucratique, commencent à porter des fruits.

L'agenda local 21 de Rio, un document extrêmement important pour cette étude met en évidence la solution des problèmes sociaux et économiques en priorité. Cette nouvelle Bible du nouveau millénaire élabore les méthodes d'égalisation des catégories antérieurement défavorisées, telles les femmes, les jeunes, les nécessiteux et leur incorporation dans la société. Toute la société engage les ressources disponibles pour adopter les principes de l'agenda 21, il faut établir un consensus, coordonner les efforts contrairement au modèle dépassé des intérêts juxtaposés. On tient compte des circonstances et des priorités locales, de l'opinion publique. On cherche la façon d'évaluer le succès ou le progrès pour estimer les résultats réels.

18 Les principes-clés sont contraires à la politique mercantile du profit, le seul paramètre du succès sans égard à son prix. Pour cette raison, l'agenda 21 est nommé nouvelle Bible car il insiste sur l'égalisation des droits de toutes les catégories sociales et s'engage en faveur de ceux sans chances réelles, des pauvres dont le nombre augmente d'un jour à l'autre. Surtout, le développement durable est considéré comme lutte contre le néolibéralisme et l'économie néo-classique - "connaitre le prix de toutes choses et la valeur de rien ». Focalisé sur l'individu, le néolibéralisme a envahi tout le monde anéantissant, tous les cadres sociaux et toutes sortes de solidarités.

19 À la Douzième Conférence Mondiale (Hong-Kong, avril 2006) sur le développement durable, l'auteur du texte a eu l'occasion de s'assurer à quel point l'idée primordiale et le principe du développement durable a pris racines dans le monde à partir des idées globales au niveau des pouvoirs locaux. Les expériences en sont surprenantes. Environ 
400 experts de par le monde ont pris part à la Conférence du Hongkong et ont exposé les expériences de leurs pays. Y participaient des architectes, des paysagistes, des hommes d'affaires, des banquiers, des écologistes, des industriels. La conférence a eu pour but de vérifier à quel point l'idée de développement durable, premièrement considérée comme illusoire, peut être incorporée dans la législation nouvelle, la pratique architecturale et son aspect social.

De même, la Conférence sur l'urbanisme corporatif (Dublin, septembre 2006.) a insisté sur la coopération de l'industrie, du commerce et de l'urbanisme aux fins communes - la solution des problèmes sociaux et de la " gentrification » de la société.

Certaines conclusions des deux conférences se référent à l'embarquement dans la société du savoir ("knowledge society ). Ceci comprend une éducation meilleure et massive. Les cours, ateliers, entrainements, acquisition des connaissances etc. et le savoir-faire offrent des possibilités a toute une pléiade de déshérités, défavorisés etc. De cette manière, la responsabilité de la société pour le chômage et la pauvreté se transfère aux individus euxmêmes.

L'action commune des experts de différents domaines, biologistes, écologistes, physiciens, architectes, urbanistes, macro-économistes, banquiers, hommes d'affaires, industriels représente une orientation approuvée du développement durable ${ }^{8}$. Tous ces experts s'engagent à créer de nouvelles « formules » et leur implantation dans la nouvelle société. On s'attend à un degré beaucoup plus élevé de solidarité. Le renouvellement urbain est reconnu comme ligne directrice du développement durable.

Tout ceci n'est, malheureusement, pour la Serbie qu'un futur très lointain. Sans une situation politique stable et au moins une décennie de donations et d'investissements des capitaux étrangers, sans une création d'emplois il est fort difficile d'atteindre le niveau prévu par les principes du développement durable. Ce qui est certainement possible c'est de faire des efforts pour retenir les jeunes experts de quitter le pays et de sauvegarder la dignité des cadres (professeurs, médecins, ingénieurs) en les payant convenablement pour leur travail de transmetteurs du savoir et leur faciliter la mise au courant des expériences et manifestations professionnelles. Un reflux des matières grises qui ne cesse en Serbie depuis 1990 est désastreux. Le développement durable recommande la société du savoir alors que la Serbie a fait fuir plus d'un million de professionnels. La Serbie souffre à présent du manque d'une génération d'âge moyen qui l'a quittée au cours des années 90, tandis que les jeunes après les études universitaires, trouvent toujours un travail mieux rémunéré en Europe, aux États-Unis, en Australie et en Nouvelle Zélande.

Les Universités fameuses d'architecture aux États-Unis ont changé leurs cours, et accordent une priorité absolue à la solution des problèmes sociaux par le moyen de l'architecture, notamment le renouvellement urbain. Les facultés en Europe, où la situation est encore plus défavorable, accèdent à cette nouvelle orientation. Bien sûr, la Serbie a envie de s'y incorporer. Cependant, l'isolement rend l'intégration dans la communauté des pays développés difficile. Les événements politiques, la situation économique sont des éléments dont on doit tenir compte en étudiant le phénomène de la rénovation urbaine. 


\section{Renouvellement urbain} des communautés locales est surtout important. La participation des représentants des agences régionales de planification et de développement est souhaitable, par exemple en Grande-Bretagne elle est assurée par « English Partnerships ». La coordination des projets d'investissement est ainsi assurée aussi bien pour le secteur public que privé et attire par une promotion créative et compréhensible la rénovation urbaine. Les trois premières agences de rénovation en Grande-Bretagne sont établies en 1999, après la publication du livre Urban Task Force Report par Lord Rogers. L'auteur propose avec beaucoup de zèle l'établissement de telles organisations dans le but d'assurer une rénovation urbaine intégrée. « English Partnerships » a depuis fondé 15 agences. Le Ministre de la rénovation, Tony McNoulty, a annoncé des fonds exceptionnels destinés à la rénovation urbaine. La rénovation effectuée sur 150 ha a assuré 4000 emplois et le rehaussement de $15 \%$ du niveau économique et social des quartiers rénovés.

29 La création des agences de rénovation urbaine (URA) est fort utile dans tous les pays. En Serbie, cette agence a été formée par l'auteur du présent texte avec des étudiants du troisième degré d'étude de la Faculté d'Architecture de l'Université de Belgrade. D'autres professeurs et experts du domaine de l'urbanisme y sont incorporés. 

dédiée exclusivement à la rénovation urbaine générale et intégrative. Des ouvrages partiels sont rénovés spontanément, selon les demandes des investisseurs du pays ou de l'étranger. La rénovation se fait sans aucun contrôle ni directive. Les investisseurs potentiels ne savent où s'adresser pour des consultations concernant les rénovations à entreprendre. En effet, leur parcours comprend l'administration de la ville, le Ministère compétent, le Secrétariat du territoire à bâtir, de l'aménagement du territoire, des contacts privés et des agences immobilières. La structure des responsabilités et des charges officielles n'est pas établie. Le problème est aggravé en Serbie par le manque de coopération de l'État (Ministère) et de l'administration locale.

31 Le processus devrait se dérouler dans le sens inverse. La ville, notamment l'État devrait, jouer le rôle de promoteur. L'offre devrait se baser sur la sélection des zones et des priorités (certaines zones pour des raisons inexplicables attendent pendant des décennies d'être rénovées). Il est important de définir rationnellement les conditions de l'offre (but, titulaires, intérêt, termes précis) pour éviter des abus et empêcher la corruption et les influences politiques ${ }^{10}$. influences des partis politiques actuellement au pouvoir. Au contraire, elles devraient définir l'attitude des pouvoirs locaux et de l'état concernant la rénovation urbaine comme facteur social. Les experts sont appelés à traiter la rénovation urbaine comme phénomène de l'entité et de compréhension et respecter les documents, projets etc. de la planification urbaine à long terme et de la prise des décisions dans un centre.

L'agence URA doit prendre une position autonome tout en collaborant étroitement, lier la ville et l'État sous leur patronage, de même avec les organes des pouvoirs locaux (Secrétariat à l'urbanisme, Institut d'urbanisme, Aménagement du territoire). Les perspectives de l'URA sont dans un avenir lointain sans égard aux changements politiques éventuels. paramètres de l'amélioration du niveau de confort et de vie dans les quartiers rénovés.

L'Agence URA, Belgrade ${ }^{11}$ fait la reconnaissance des localités et des quartiers potentiels selon les paramètres et les priorités établis, présente des "swot» analyses (sans de pareils documents l'investisseur ne peut envisager d'achat foncier). Elle s'engage dans le processus de la délivrance des permis prioritaires en collaboration avec les organes des pouvoirs locaux. A la demande de l'investisseur l'Agence assure l'élaboration :

- du projet,

- de la construction financière préliminaire,

- des contrats,

- du marketing,

- assure la gestion des travaux.

L'URA étudie les possibilités du développement économique du site, suggère les directions et les méthodes de rénovation, l'équipement du site en infrastructure, les technologies disponibles du site, les ressources humaines adaptables et motivées sur le site (et comment poursuivre leur amélioration après la rénovation). L'URA dresse un nouvel aspect du site selon la stratégie du «flagship» et résout à long terme les «brownfields ». Le groupe visé est la population défavorisée dont la situation économique et l'habitat seront améliorés par la rénovation et les nouveaux emplois assurés. 
L'organisation de l'Agence au moment de sa création ne put être réalisée que à titre de rassemblement des citoyens (ONG). Une coopération directe de l'État, de la ville, des experts et du secteur privé est une pratique favorisée en cas d'une activité semblable. En dépit des efforts déployés pour établir un support réel et non seulement officiel du Gouvernement et des pouvoirs locaux, l'Agence n'a pas connu de volonté politique pour sa promotion active. Les pourparlers sont en cours avec l'» English Partnerships » intéressé à fournir une aide technique.

On s'attend à ce que Belgrade comprenne les avantages des activités de l'Agence URA, en premier lieu pour résoudre les problèmes d'un grand nombre de personnes sans abri et défavorisées. Les intérêts personnels sont à bannir lorsqu'il s'agit d'une tâche aussi délicate. L'expérience acquise dans d'autres pays prouve qu'un groupe de jeunes gens guidés et contrôlés par les meilleurs experts et par les pouvoirs respectifs, peut mener la rénovation mieux qu'une bureaucratie encombrante datant de l'époque de l'exYougoslavie (5 fois plus grande que la Serbie).

\section{Protection des personnes défavorisées grâce à la rénovation des immeubles collectifs}

39 Une innovation absolue dans le domaine de la rénovation urbaine vise à résoudre des problèmes sociaux comme l'affectation des immeubles collectifs abandonnés des années 50 et 60 - à l'hébergement des personnes défavorisées et leur reconstruction.

Ces entreprises sont effectuées dans l'esprit des principes du développement durable. Il s'agit des bâtiments existants de propriété publique, ce qui facilite l'opération. Les normes de confort de ces immeubles collectifs des grands ensembles ne sont plus satisfaisantes même pour le logement social. Ils nécessitent un "refurnishment» comprenant la rénovation de l'équipement et de l'infrastructure. Parfois ces logements sont agrandis et reconstruits. Étant donné qu'actuellement le standing des logements est de plus en plus élevé, de nombreux logements des années 50 et 60 ne correspondent plus aux critères techniques et fonctionnels et ne sont pas pourvus d'équipements satisfaisants. Belgrade dispose de blocs d'immeubles collectifs dans lesquels on n'a rien investi durant cinquante ans. En plus il y a des constructions industrielles qui se prêtent au recyclage urbain et à l'affectation au logement social pour toute une série de personnes démunies et défavorisées.

41 Les sans-abri de Belgrade, les invalides de guerre, les personnes défavorisées, exigent des solutions rapides. Le renouvellement urbain ne donne pas de résultats immédiats et c'est son inconvénient grave. En plus, l'État ne dispose pas de fonds pour satisfaire les besoins de toutes les catégories de nécessiteux, une aide des pays étrangers serait nécessaire. De nombreux pays ont témoigné une solidarité exemplaire envers la Serbie. Cependant, les donations étrangères arrivent à leur fin, et il ne reste qu'à faire appel à ses propres ressources.

\section{Conclusion}

Le renouvellement urbain est utile et applicable en Serbie au moment où celle-ci se trouve dans une situation socio-économique défavorable. La situation est considérée dans l'optique de nouvelles caractéristiques sociales. Autrefois on croyait que le patrimoine 
architectural devrait rester intact ; à présent on sait bien que ce n'est pas possible et que le principe de "protection active» est à respecter. Le nouveau tissu urbain doit être incorporé dans le tissu hérité, celui-ci doit subir des améliorations, doit être activé et doit développer tous ses potentiels disponibles. Le renouvellement des villes est traité dans des circonstances socio-économiques défavorables. L'écologie et le recyclage urbain deviennent des éléments incontournables de l'urbanisme. La Serbie s'adapte lentement aux orientations européennes, consciente que pendant un bon moment elle ne pourra s'incorporer ni économiquement ni autrement dans les courants européens, ni suivre les modèles prestigieux.

La coordination des activités de renouvellement urbain est d'une importance primordiale, l'interaction de la ville, de l'État et des donateurs est obligatoire pour pourvoir aux besoins de la nouvelle situation socio-économique. Le service social et la préoccupation pour la santé et les besoins des citoyens sont au premier plan. Ce n'est qu'aujourd'hui, malheureusement, lorsque l'on a compris que ni la santé publique, ni l'éducation ne peuvent être gratuits ni les HLM abordables pour les démunis, que nous sommes conscients de la distance à parcourir pour atteindre l'exemple de la politique sociale de Hong-Kong ou de la Norvège ; Hong-Kong profite de la rencontre du capitalisme et du socialisme; le premier fournit les fonds (capital du commerce et bancaire), la politique sociale du deuxième sert de modèle. La santé publique et l'éducation gratuite en résultent. La Norvège est, grâce aux ressources provenant du pétrole, le pays européen le plus avancé en politique sociale.

Dans le cadre de l'ex-Yougoslavie et de l'ordre socialiste, la Serbie jouissait d'une situation économique stable et d'une politique sociale des plus avancées basée, malheureusement, sur des crédits et des dettes extérieures. Pour cette raison, la population âgée et d'âge moyen s'adapte difficilement aux rudes circonstances capitalistes qui donnent peu de chance aux groupes défavorisés (démunis, âgés, génération d'âge moyen, minorités, etc.).

La nouvelle méthode de faire usage des immeubles abandonnés et de les rénover en HLM est une des modalités de la lutte pour la solution de graves problèmes sociaux. Ceci demande le savoir des experts, une organisation peu nombreuse et flexible, l'interaction de la Ville, de l'État et du Donateur, la bonne volonté des personnages politiques et l'élimination de la corruption. Une nouvelle législation est nécessaire visant à la rationalisation des problèmes administratifs et bureaucratiques et à la socialisation de la régulation législative.

$\mathrm{Vu}$ que la Serbie ne peut pas compter sur une aide financière continue il serait fort nécessaire de procéder à la création de nouveaux postes de travail selon ses propres potentiels. Une activité dans le domaine du renouvellement urbain et de la planification représente un des potentiels prometteurs. Au cours des années précédentes, les donations affluaient mais les projets manquaient. À présent il faut avoir recours aux ressources humaines et financières du pays. Une approche globale est à adopter ; la coordination des activités jusqu'à présent le plus souvent isolées est une des recommandations du développement durable.

47 Un État stable est un préalable de l'urbanisme « investisseur » (strictement régularisé par les lois, les directives et les besoins du développement y compris tous les effets sur les constituants macroéconomiques), qui pourrait actuellement compenser le retard du pays. 
Les activités de l'Agence de renouvellement sont des voies possibles pour améliorer les relations entre l'État et les urbanistes vers la « gentrification » des villes.

\section{BIBLIOGRAPHIE}

CUPEM 20th International Conference, Re-inventing Global Cities, The Centre of Urban Planning and Environmental Management, Hong Kong, 2000.

Economic Commission for Europe, Country Profiles on the Housing Sector, Serbia and Montenegro, United nations, New York, Genève, 2006.

The 12th Annual International Sustainable Development Research Conference 2006, 6-8 April 2006, Hong Kong Convention \& Exhibition Centre, University of Hong Kong, 2006.

International Journal of Innovation \& Sustainable Development, vol.1, n. 1/2, Genève, 2005.

Prokopijević M., “Evropska Unija, socijalna politika”, Službeni glasnik, Belgrade, 2005.

Progress in Industrial Ecology, An international Journal, vol. 1, nos. 1/2/3,Genève, 2004.

Urbanisme, n. 338, 337, 336, 334 et hors-série n. 21, 20, Paris, 2004.

Vaništa Lazarević E., „Urbana rekonstrukcija“, Zadužbina Andrejević, Belgrade, 1999.

Vaništa Lazarević E., „Obnova gradova u novom milenijumu“, Classic Map Studio, Belgrade, 2002.

Vaništa Lazarević E., „Urbana obnova, četvrt veka posle Londonskih dokova“, (sous presse).

Zbornik, „Za bolji put Srbije“, Demokratska stranka, centar resornih odbora, Belgrade, 2006.

\section{NOTES}

1. Comité européen pour la cohésion sociale, Approche des droits sociaux en Europe, Strasbourg, 2002.

2. M. Prokopijević, Union européenne, politique sociale, Belgrade, 2003.

3. D'après Commission d'économie de l'Europe/ONU, Habitat, p. 3.

4. D'après le Ministère de la politique Sociale, documentation interne.

5. Le parti Démocrate, Une Serbie meilleure, Belgrade, 2006.

6. UNHCR 2004.

7. http://www.sustainableworld.com

8. Revue internationale de l'innovation et du développement durable, Genève, 2006.

9. E. Vaništa Lazarević, Le renouvellement urbain à l'aube du nouveau Millénaire, Belgrade, 2002.

10. Documents internes, Agence de renouvellement urbain, Belgrade.

11. L'Agence est crée en 2004, avec le cadre juridique d'une ONG, avec le support des pouvoirs locaux de Belgrade, de la SIEPA (Agence pour la promotion des exportations), de la Faculté d'Architecture et de l'Association des urbanistes de Belgrade. 


\section{RÉSUMÉS}

L'auteur dresse le tableau administratif et législatif de l'urbanisme à Belgrade au début des années 2000, en le comparant à celui d'autres pays. Elle propose une amélioration du tissu hérité qui intègre une réflexion écologique, ainsi qu'une meilleure coordination entre l'état, les donateurs et les communautés locales.

The author gives an administrative and legal overview of urbanism in Belgrade at the beginning of the 2000's in comparison with other countries. For her, it would be convenient to improve inherited substrate in integrating ecology constraints, and to coordinate State, donators and local communities.

\section{AUTEUR}

\section{EVA VANIŠTA LAZAREVIĆ}

Université de Belgrade 